\title{
The RANKL/RANK/OPG signal trail: significance of genetic polymorphisms in the etiology of postmenopausal osteoporosis
}

\author{
Hubert Wolski ${ }^{1,2}$, Krzysztof Drews ${ }^{1,3}$, Anna Bogacz ${ }^{4,5}$, Adam Kamiński ${ }^{6}$, Magdalena Barlik ${ }^{1,3}$ \\ Joanna Bartkowiak-Wieczorek4, Andrzej Klejewski ${ }^{7,8}$, Marcin Ożarowski ${ }^{5,9}$, \\ Marian Majchrzycki ${ }^{10}$, Agnieszka Seremak-Mrozikiewicz ${ }^{1,3,5}$ \\ ${ }^{1}$ Division of Perinatology and Women's Diseases, Poznan University of Medical Sciences, Poznan, Poland \\ ${ }^{2}$ Division of Gynecology and Obstetrics, Podhale Multidisciplinary Hospital, Nowy Targ, Poland \\ ${ }^{3}$ Laboratory of Molecular Biology in Division of Perinatology and Women's Diseases, \\ Poznan University of Medical Sciences, Poznan, Poland \\ ${ }^{4}$ Laboratory of Experimental Pharmacogenetics, Department of Clinical Pharmacy and Biopharmacy, \\ University of Medical Sciences, Poznan, Poland \\ ${ }^{5}$ Department of Pharmacology and Phytochemistry, Institute of Natural Fibers and Medicinal Plants, Poznan, Poland \\ ${ }^{6}$ Department of Pediatric Orthopaedics and Traumatology, Pomeranian Medical University, Szczecin, Poland \\ ${ }^{7}$ Department of Nursing, Poznan University of Medical Sciences, Poznan, Poland \\ ${ }^{8}$ Department of Obstetrics and Women's Diseases, Poznan University of Medical Sciences, Poznan, Poland \\ ${ }^{9}$ Department of Pharmaceutical Botany and Plant Biotechnology, Poznan University of Medical Sciences, Poznan, Poland \\ ${ }^{10}$ Department of Rheumatology and Rehabilitation, Poznan University of Medical Sciences, Poznan, Poland
}

\begin{abstract}
Objectives: Recent studies have demonstrated that disorders of bone metabolism, which is regulated by RANK/RANKL/OPG signaling pathway, are the cause of osteoporosis. The aim of the study was to investigate the distribution of genotypes of the RANK $575 C>T$ and RANKL $-643 C>T$ polymorphisms and to analyze their relationship with bone parameters in postmenopausal women.

Material and methods: A total of 310 postmenopausal Caucasian women (139 with osteoporosis, 107 with osteopenia, and 64 healthy postmenopausal controls) were included. Bone mineral density (BMD) at the lumbar region of the spine (L2-L4) was measured by dual energy X-ray absorptiometry (DXA). Genetic analysis was performed using the PCR-RFLP method. Results: Analysis of the frequency of genotypes and alleles of the RANK 575C >T and RANKL $-643 C>T$ polymorphisms did not show any statistically significant differences between the study groups (osteoporosis and osteopenia) and postmenopausal women with normal $t$-score value (ns). Notably, a significant association between the RANKL $-643 C>T$ polymorphism and body mass, such as BMI values in osteoporotic women $(p<0.05)$, was observed.

Conclusions: Our results suggest lack of association between the 575C > RANK polymorphism and the development of osteoporosis. The $-643 C>T$ RANKL polymorphism, through its significant influence on body weight and BMI value, may contribute to the development of osteoporosis in postmenopausal women.
\end{abstract}

Key words: osteoporosis, RANKL/RANK/OPG signaling trail, genetic polymorphism

Ginekologia Polska 2016; 87, 5: 347-352

Corresponding author:

Agnieszka Seremak-Mrozikiewicz

Division of Perinatology and Women's Diseases, Poznan University of Medical Sciences, Polna 33, 60-535 Poznan, Poland

e-mail:asm@data.pl 


\section{INTRODUCTION}

Osteoporosis is a serious metabolic disorder of old age, characterized by decreased bone mineral density. Osteoporosis is a complex disease and numerous studies have demonstrated that hormonal, environmental, and genetic factors play a significant role in its etiology [1-4]. Osteoporosis is a vital problem among elderly populations. Disturbances in bone microarchitecture metabolism often lead to bone fractures, restricted mobility, and death, making osteoporosis a medical as well as socio-economical problem $[5,6]$. Thus, numerous etiological studies are focused on factors involved in the regulation of bone mineralization. Several of these factors are involved in the process of impaired osteoclastic activity and increased bone resorption. Their observations highlight the significance of the contribution of genetic polymorphisms to osteoclastic activity $[7,8]$.

In recent years, it has been noted that the processes of growth, maturity, segregation and differentiation of osteoclasts are synchronized and modulated by various factors, with the signal pathway, which is directly involved in bone resorption, playing the key role $[9,10]$. Currently, it is believed that the receptor activator of nuclear factor kappa B (RANK), its ligand (RANKL - receptor activator of nuclear factor-KB ligand) and osteoprotegerin (OPG) are involved in this trail $[9,10]$. The abovementioned factors belong to the tumor necrosis factor (TNF) family. It seems that dynamic steadiness between RANKL/RANK and OPG activities determines regulation of differentiation and activation of osteoclasts, significantly affecting bone tissue metabolism. The RANKL/RANK/OPG trail is directly involved in proliferation and apoptosis of osteoclasts. The disturbances in the RANKL/RANK/OPG system can be a major part of the etiology of postmenopausal osteoporosis. Moreover, it has been shown that genes encoding RANKL/RANK/OPG system are closely involved in the development of osteoporosis [10, 11].

The RANKL protein is the key factor for separation and demarcation of osteoclasts, responsible for their enrolment, fusion into multinuclear osteoclasts, and activation of the mature forms. Another major function of RANKL is the inhibition of osteoclast apoptosis [11]. The transmembrane RANK protein is the receptor of RANKL. Results of the RANK/RANKL fusion include differentiation of preosteoclasts into mature forms, increase of osteoclasts activity, and inhibition of osteoclastic apoptosis [9]. The third factor in the described pathway is osteoprotegerin, a dimeric, soluble cytokine produced in the activated osteoblasts. OPG is a 'receptor-trap' for RANKL and, in consequence, inhibits the entire trail of osteoclast differentiation at the early stages. In this mechanism, OPG is proposed to play the role of a bone resorption inhibitor, as well as the factor of bone mass regulating. OPG suppresses osteoclast differentiation, blocks their activity, and induces osteoclast apoptosis [12]. The most important function of OPG is reduction of the number of active osteoclasts and, through this process, inhibition of bone resorption. Some genetic polymorphisms in the RANKL/RANK/OPG signal trail have been determined. Several polymorphic variants of these genes have been postulated to be connected with osteoporosis in postmenopausal women [13-17].

The aim of the study was to examine the frequency of alleles and genotypes of the RANK 575C $>T$ and RANKL $-643 C>T$ polymorphisms in a group of postmenopausal women and to determine the association of the examined genetic variants with parameters of bone turnover and progression of osteoporosis.

\section{MATERIAL AND METHODS}

The study (conducted between 2004-2006) included 310 postmenopausal Caucasian women ( $54.5 \pm 8.5$ years). The patients were classified into three subgroups with regard to the value of the rate $t$-score: 139 with osteoporosis ( $t$-score $<-2.5,56.1 \pm 8.8$ years), 107 with osteopenia ( $t$-score between 2.5 and $-1,53.3 \pm 8.1$ years), and 64 healthy postmenopausal controls ( $t$-score $>-1,53.4 \pm 8.2$ years of age). Menopause onset at least one year before the study constituted an eligibility criterion. The exclusion criteria were as follows: history of double-sided ovariectomy, medications which might influence bone metabolism (hormone therapy, selective modulators of estrogen receptors, thyrocalcitonin, bisphosphonates, thyroid hormones, steroids, heparin, anticonvulsant medications, GnRH analogues, tibolone), and history of diseases affecting bone density and loss of bone mass (endocrine and metabolic disorders, hematological diseases, cancers, nephropathies and autoimmune diseases). On the basis of densitometry tests (DXA — dual energy X-ray absorptiometry, LUNAR DPX 100 apparatus, Lunar Corp., Madison, USA), the following parameters describing the state of bone tissue were evaluated: bone mineral density (BMD) in the lumbar region of the spine (L2-L4), as well as the rates: $t$-score and $z$-score. Rates of medium bone mineral density were also evaluated in comparison to the average for young adults (YA - young adults) and the average bone mineral density in comparison to the average for the given age (AM - age matched). Additionally, body mass index (BMI) was determined on the basis of body weight and height measurements. Local Ethics Committee approved of the study (1426/04). All women gave their written informed consent.

The RANK 575C $>T$ and RANKL $-643 C>T$ polymorphisms were determined by polymerase chain reaction/restriction fragment length polymorphism (PCR/RFLP) analysis. For the analysis of the RANK 575C $>$ T and RANKL $-643 C>T$ polymorphisms, the following primers were used: RANK-forward: 5'- CAT CAT GGG ACA GAG AAA TCC GAC G -3', RANK-reverse: 5'- GAG AAT GAA ATA TTC AGC TGA CC -3', RANKL: 5'- GGA 
TGC TTG CTT CTG GCT AC -3', RANKL: 5'- ACC CTT CCT GTC CAA CCT CT -3'. The polymorphic sites were defined using restriction enzymes for the RANK gene, such as BstUl and TscAl for RANKL. Products of the electrophoresis were evaluated using visualization with UV light, using the system of documentation and computer analysis of the UVI image (KS 4000/Image PC of the Syngen Biotech Molecular Biology Instruments company).

SPSS 17.0 PL software was used for statistical analysis of the results of the frequency of genotypes and alleles of the examined polymorphisms. The prevalence of certain genotypes was compared with the expected values using the chi-square test. The analysis of the clinical and biochemical parameters and their relationship with the previously reported polymorphisms was performed using one-way ANOVA test (SPSS Inc.). The $p$-value of $<0.05$ was considered as statistically significant.

\section{RESULTS}

Analysis of the frequency of genotypes and alleles of the RANK 575C $>T$ and RANKL $-643 C>T$ polymorphisms did not show any statistically significant differences between the study groups (osteoporosis and osteopenia) as compared to postmenopausal women with normal $t$-score values (ns) (Table 1).

Moreover, average parameters of bone turnover for each genotype of the examined RANK $575 C>T$ and RANKL $-643 C>T$ polymorphisms have been also compared between the investigated subgroups. No significant correlations between the majority of the parameters of bone turnover and frequency of particular genotypes in women with osteoporosis, osteopenia and postmenopausal women with normal $t$-score values have been observed (Table 2). Noteworthy, a significant association between the RANKL $-643 C>T$ polymorphism and body mass, such as BMI values in osteoporotic women, was observed $(p<0.05)$ (Table 2).

\section{DISCUSSION}

Diagnostic testing for the presence of genetic polymorphisms, or their influence on the values of parameters determining the state of bone tissue in women after menopause, is essential to understand the pathomechanism of osteoporosis. The exact effect of the RANKL $-643 C>T$ and RANK $575 C>T$ polymorphisms on bone mineral density remains vague. Assessment of the impact of OPG gene polymorphisms on bone turnover parameters is also essential. Many studies focus on the correlation of the RANK/RANKL/OPG signaling pathway polymorphisms with bone mineral density and other parameters $[13,16,18]$.

In our recent study, we analyzed the significance of $-163 A>G,-254 T>G,-950 T>C$ and $1181 \mathrm{G}>C$ polymorphisms in the development of osteoporosis. No statistically significant differences in the examined subgroups of women with osteoporosis, osteopenia and correct values of t-score have been shown [17].

The present study, performed in a large population of Polish women, also did not show any correlation between the investigated polymorphisms and BMD values. We investigated other polymorphism in the RANK/RANKL/OPG signaling pathway in connection with the pathomechanism

Table 1. Frequency of genotypes and alleles of RANK and RANKL polymorphisms in women with osteopenia, osteoporosis and normal t-score values

\begin{tabular}{|c|c|c|c|c|c|c|c|}
\hline & & \multicolumn{2}{|c|}{$\begin{array}{l}\text { Osteoporosis } \\
n=139\end{array}$} & \multicolumn{2}{|c|}{$\begin{array}{l}\text { Osteopenia } \\
n=107\end{array}$} & \multicolumn{2}{|c|}{$\begin{array}{l}\text { Normal t-score value } \\
\qquad n=64\end{array}$} \\
\hline & & $\begin{array}{l}\text { Observed } \\
\text { n (\%) }\end{array}$ & $\begin{array}{l}\text { Expected } \\
(\%)\end{array}$ & $\begin{array}{l}\text { Observed } \\
\text { n (\%) }\end{array}$ & $\begin{array}{l}\text { Expected } \\
\qquad \%)\end{array}$ & $\begin{array}{l}\text { Observed } \\
\text { n (\%) }\end{array}$ & $\begin{array}{l}\text { Expected } \\
\text { (\%) }\end{array}$ \\
\hline \multirow{6}{*}{$\begin{array}{c}\text { RANK } \\
575 C>T\end{array}$} & $\mathrm{CC}$ & 47 (33.8) & 33.5 & $22(20.6)$ & 23.6 & $17(26.6)$ & 26.6 \\
\hline & CT & $67(48.2)$ & 48.8 & $60(56.1)$ & 50.0 & $32(50.0)$ & 49.9 \\
\hline & TT & 25 (18.0) & 17.7 & 25 (23.4) & 26.4 & $15(23.4)$ & 23.4 \\
\hline & Allele & & & & & & \\
\hline & $C$ & $161(57.9)$ & - & $104(48.6)$ & - & 66 (51.6) & - \\
\hline & $\mathbf{T}$ & $117(42.1)$ & - & $110(51.4)$ & - & $62(48.4)$ & - \\
\hline \multicolumn{8}{|c|}{ Genotype } \\
\hline \multirow{6}{*}{$\begin{array}{c}R A N K L \\
-643 C>T\end{array}$} & CC & 33 (23.7) & 26.4 & 20 (18.7) & 21.8 & $13(20.3)$ & 24.2 \\
\hline & CT & 77 (55.4) & 49.9 & $60(56.1)$ & 49.8 & $37(57,8)$ & 50.0 \\
\hline & TT & $29(20.9)$ & 23.6 & $27(25.3)$ & 28.4 & 14 (21.9) & 25.8 \\
\hline & Allele & & & & & & \\
\hline & C & $143(51.4)$ & - & $100(46.7)$ & - & 63 (49.2) & - \\
\hline & $\mathbf{T}$ & 135 (48.6) & - & $114(53.3)$ & - & $65(50.8)$ & - \\
\hline
\end{tabular}




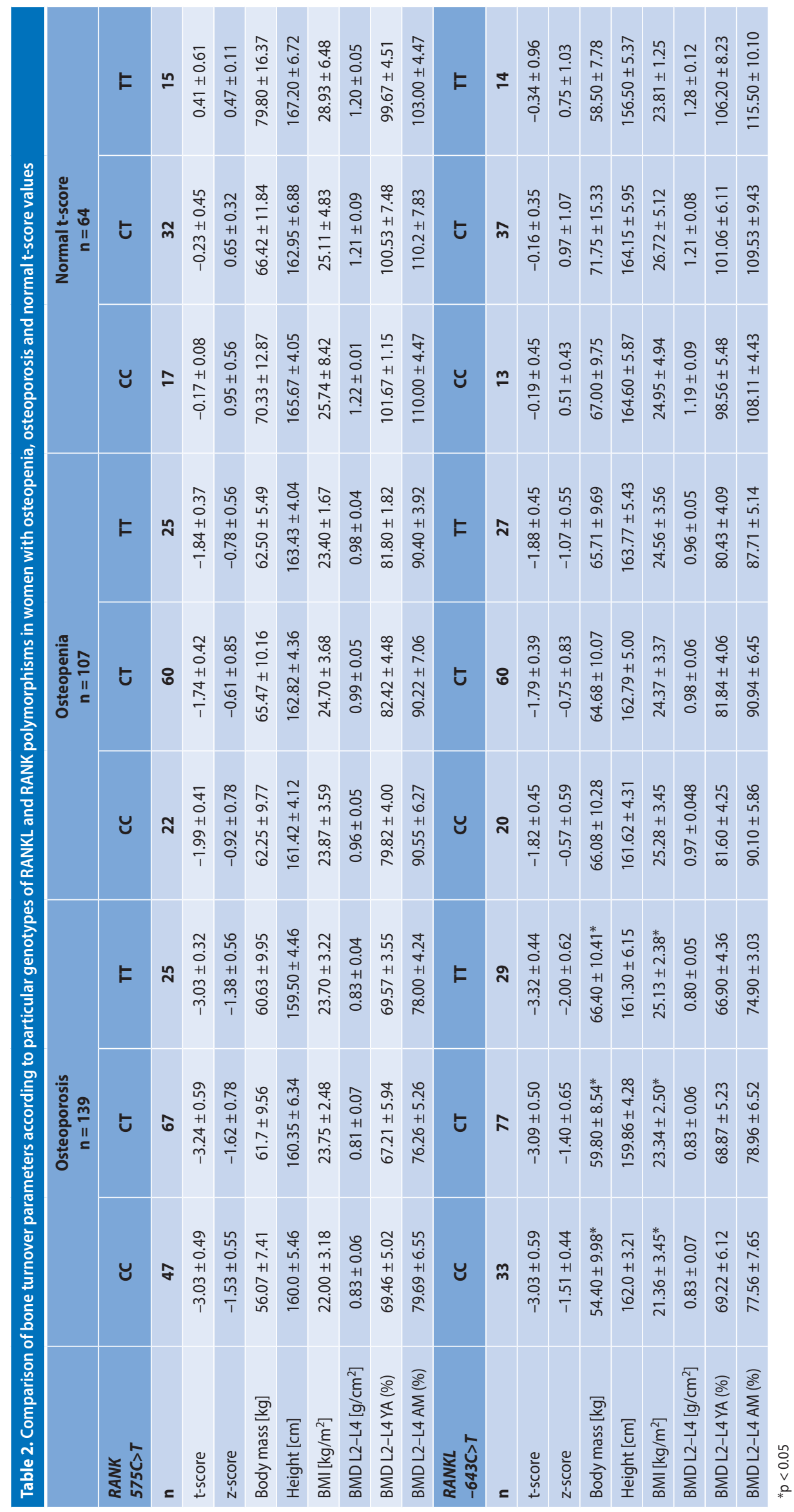


of osteoporosis. The genotype frequency of RANKL $-643 C>T$ and RANK $575 C>T$ polymorphisms was similar to the distribution of genotypes in other Caucasian populations [19-21]. Analysis of both investigated RANKL/RANK polymorphisms revealed no significant association between particular genotypes and osteoporosis, or development of osteopenia. Additionally, for the RANKL $-643 C>T$ and RANK $575 C>T$ polymorphisms, no statistically significant correlations between the studied genotypes, development of osteoporosis, and bone parameters such as t-score, z-score, L2-L4 BMD YA\% or L2-L4 BMD AM\% were noted in all groups of women. Interestingly, for the RANKL $-643 C>T$ polymorphism we showed a statistically significant relationship between the CC genotype and lower body weight and BMI.

Similar results to our findings were reported in a study of postmenopausal women in Slovenia, which also confirmed no relationship between bone mineral density in the lumbar spine and hip BMD and the $-643 C>T$ polymorphism. However, higher BMD values in the femur neck were noted in TT genotype carriers as compared to CC carriers [19]. There were no statistically significant differences between genotype frequency in the group of healthy women and women with osteoporosis. A significant correlation between all genotypes and bone mineral density in the lumbar spine $(p=0.041)$ has been demonstrated in the group of women with osteoporosis, whereas there was no association between genotypes and BMD of the femoral neck (BMD-fn) and total femur BMD (BMD-th). The analysis of healthy women showed no relationship between the particular genotypes of the $-643 C>T$ polymorphism and bone mineral density in the lumbar spine (BMD-Is), bone mineral density of the femoral neck (BMD-fn), and total femoral bone mineral density (BMD-th) [20].

Distribution of the RANK 575C $>T$ polymorphism differs in the investigated populations and the results obtained among Polish women were significantly different as compared to Korean or Chinese populations. The influence of the RANK $575 C>T$ polymorphism on bone parameters remains unclear. Results referring to the Polish population also have shown that the RANK575C > T polymorphism did not seem to be a genetic factor associated with BMD of the lumbar spine, similarly to Korean postmenopausal women [22]. A study conducted in an Asian population of postmenopausal women showed that the 575C $>T$ RANK polymorphism is not associated with BMD of the lumbar spine and femoral neck [22-24].

In a Chinese population, patients with low BMD were compared to individuals with high BMD to show a link between the evaluated polymorphisms and BMD [25]. Studies of the Chinese population demonstrated the relations of one of the RANK polymorphisms with BMD of the lumbar spine and hip joint [26]. The same authors showed that the RANK and RANKL genes were associated with age of first menarche as well as age at natural menopause in Caucasian females in the United States [26].

Despite numerous genetic analyses, contribution of the polymorphic variants to the development of osteoporosis remains unclear. Studies evaluating a possible correlation between the RANK and RANKL polymorphisms and bone mineral density as well as the development of osteoporosis also offered no conclusive results. Thus, large, multi-center studies are necessary to assess the distribution of the RANKL and RANK polymorphic variants in Caucasian populations.

\section{CONCLUSIONS}

Our results suggest lack of a association between the $575 C>T$ RANK polymorphism and the development of osteoporosis, whereas the $-643 C>T$ RANKL polymorphism, due to its significant influence on body weight and BMI values in postmenopausal women, could contribute to the development of osteoporosis.

\section{REFERENCES}

1. Rachner TD, Khosla S, Hofbauer LC. Osteoporosis: now and the future. Lancet. 2011, 377, 1276-1287.

2. Ferrari S. Human genetics of osteoporosis. Best Pract Res Clin Endocrinol Metab. 2008, 22, 723-735.

3. Garcia-Unzueta MT, Rancho JA, Zarrabeltia MT, [et al.]. Association of the $163 \mathrm{~A} / \mathrm{G}$ and $1181 \mathrm{G} / \mathrm{C}$ osteoprotegerin polymorphism with bone mineral density. Horm Metab Res. 2008, 40, 219-224.

4. Wolski H, Drwęska-Matelska N, Seremak-Mrozikiewicz A, [et al.]. The role of Wnt/ $\beta$-catenin pathway and LRP5 protein in metabolism of bone tissue and osteoporosis etiology. Ginekol Pol. 2015, 86, 311-314.

5. Khosla S. Pathogenesis of age-related bone loss in humans. J Gerontol A Biol Sci Med Sci. 2013, 68, 1226-1235.

6. Nachtigall MJ, Nazem TG, Nachtigall RH, [et al.]. Osteoporosis risk factors and early life-style modifications to decrease disease burden in women. Clin Obstet Gynecol. 2013, 56, 650-653.

7. Nguyen TV, Eisman JA. Genetics and the individualized prediction of fracture. Curr Osteoporos Rep. 2012, 10, 236-244.

8. Brandi ML. An overview of osteoporosis: from genetics to clinics. Aging Clin Exp Res. 2011, 23, 3-5.

9. Uemura $\mathrm{H}$, Yasui T, Miyatani $Y$, [et al.]. Circulating profiles of osteoprotegerin and soluble receptor activator of nuclear factor kappaB ligand in postmenopausal women. J Endocrinol Invest. 2008, 31, 161-168.

10. Rogers A, Eastell R. Circulating osteoprotegerin and receptor activator for nuclear factor kappaB ligand: clinical utility in metabolic bone disease assessment. J Clin Endocrinol Metab. 2005, 90, 6323-6331.

11. Raisz LG. Pathogenesis of osteoporosis: concepts, conflicts, and prospects. J Clin Invest. 2005, 115, 3318-3325.

12. Trouvin AP, Goëb V. Receptor activator of nuclear factor-kB ligand and osteoprotegerin: maintaining the balance to prevent bone loss. Clin Interv Aging. 2010, 5, 345-354.

13. Paternoster L, Ohlsson C, Sayers A, [et al.]. OPG and RANK polymorphisms are both associated with cortical bone mineral density: findings from a metaanalysis of the Avon longitudinal study of parents and children and gothenburg osteoporosis and obesity determinants cohorts. J Clin Endocrinol Metab. 2010, 95, 3940-3948.

14. Shang M, Lin L, Cui H. Association of genetic polymorphisms of RANK, RANKL and OPG with bone mineral density in Chinese peri- and postmenopausal women. Clin Biochem. 2013, 46, 1493-1501.

15. Urano T, Inoue S. Genetics of osteoporosis. Biochem Biophys Res Commun. 2014, 452, 287-293.

16. Guo L, Tang K, Quan Z, [et al.]. Association between seven common OPG genetic polymorphisms and osteoporosis risk: a meta-analysis. DNA Cell Biol. 2014, 33, 29-39.

17. Seremak-Mrozikiewicz A, Barlik M, Drews K, [et al.]. The genetic variants of RANKL/RANK/OPG signal trial in postmenopausal women with osteopenia and osteoporosis. Arch Perinatal Med. 2011, 17, 72-80. 
18. Choi JY, Shin A, Park SK, [et al.]. Genetic polymorphisms of OPG, RANK, and ESR1 and bone mineral density in Korean postmenopausal women. Calcif Tissue Int. 2005, 77, 152-159.

19. Mencej S, Albagha OM, Prezelj J, [et al.]. Tumour necrosis factor superfamily member 11 gene promoter polymorphisms modulate promoter activity and influence bone mineral density in postmenopausal women with osteoporosis. Mol Endocrinol. 2008, 40, 273-279.

20. Mencej S, Prezelj J, Kocijancic A, [et al.]. Association of NFSF11 gene promoter polymorphisms with bone mineral density in postmenopausal women. Maturitas. 2006, 55, 219-226.

21. Takacs I, Lazary A, Kosa JP, [et al.]. Allelic variations of RANKL/OPG signaling system are related to bone mineral density and in vivo gene expression. Eur J Endocrinol. 2010, 162, 423-431.

22. Kim JG, Kim JH, Kim JY, [et al.]. Association between osteoprotegerin (OPG), receptor activator of nuclear factor-KB (RANK), and RANK ligand (RANKL) gene polymorphisms and circulating OPG, soluble RANKL levels, and bone mineral density in Korean postmenopausal women. Menopause. 2007, 14, 913-918.

23. Koh JM, Park BL, Kim DJ, [et al.]. Identification of novel RANK polymorphisms and their putative association with low BMD among postmenopausal women. Osteoporos Int. 2007, 18, 323-331.

24. Zupan J, Mencej-Bedrac S, Jurkovic-Mlakar S, [et al.]. Gene-gene interactions in RANK/RANKL/OPG system influence bone mineral density in postmenopausal women. J Steroid Biochem Mol Biol. 2010, 118, 102-106.

25. Hsu YH, Niu T, Terwedow HA, [et al.]. Variation in genes involved in the RANKL/RANK/OPG bone remodeling pathway are associated with bone mineral density at different skeletal sites in men. Hum Genet. 2006, 118, 568-577.

26. Liu JM, Zhang MJ, Zhao L, [et al.]. Analysis of recently identified osteoporosis susceptibility genes in Han Chinese Women. J Clin Endocrinol Metab. 2010, 95, 112-120. 